Classification

Physics Abstracts

78.20Bh $-78.65 \mathrm{Pi}-71.10+\mathrm{x}$

\title{
Une modélisation de l'influence de la métallisation des objets et de la forme des sondes en SNOM
}

\author{
Dominique Barchiesi et Daniel Van Labeke \\ Laboratoire d'Optique P.M. Duffieux, URA CNRS 214, Université de Franche- Comté, Route de \\ Gray, 25030 Besançon Cedex, France
}

(Reçu le 20 août 1993; accepté le 15 octobre 1993)

\begin{abstract}
Résumé. - Depuis quelques années, diverses techniques de microscopie en champ proche (SNOM : Scanning Near-Field Optical Microscopy) permettent d'obtenir des images avec une résolution latérale bien meilleure que le critère dè Rayleigh $(\lambda / 30 \approx 20 \mathrm{~nm}$ sont annoncés). Les images obtenues sont très dépendantes des conditions expérimentales (angle d'incidence, polarisation, orientation du plan d'incidence, nature de l'objet...) Des études théoriques sont encore nécessaires pour modéliser les divers microscopes et interpréter les images. Nous proposons un modèle basé sur une description macroscopique de l'objet et de la sonde et utilisant une résolution perturbative des équations de Maxwell. Il s'agit ici d'une extension des travaux précédents à des objets complexes (multicouches) et des sondes de forme plus générale. De plus, le couplage électromagnétique entre la sonde et l'objet distants de quelques nanomètres est maintenant prise en compte.
\end{abstract}

\begin{abstract}
Recently, various versions of Scanning Near-Field Optical Microscopes (SNOM) have been developed, pushing the limits of lateral resolution beyond the Rayleigh criterion: $\lambda / 30 \approx 20 \mathrm{~nm}$ have been reached. Unfortunately, images obtained by SNOM depend strongly on the experimental conditions (angle of incidence, polarization, incidence plane direction, sample nature...) Theoretical studies are thus necessary for interpreting experimental data. In a previous work, we proposed a model based on a perturbative resolution of Maxwell equations. Here, we extend this work to more complex samples (multilayers) and to more general tip geometries. Moreover, this model can be used for various SNOM configurations and take the sample-tip electromagnetic coupling into account.
\end{abstract}

\section{Introduction}

Parmi les microscopies à sonde locale, les microscopies optiques en champ proche, (Scanning Near field Optical Microscope : SNOM) atteignent aujourd'hui une résolution latérale d'environ $20 \mathrm{~nm}$ [1-4] soit environ $\lambda / 30$. Cette résolution est bien meilleure que celle donnée par le critère de Rayleigh qui limite le pouvoir séparateur des microscopes traditionnels en champ lointain. Divers dispositifs expérimentaux ont été proposés, la sonde est soit une fibre optique taillée en pointe, soit une très petite ouverture dans un écran métallique. L'objet peut être éclairé par une 
onde plane en réflexion externe ou en réflexion totale interne. Il s'agit alors d'un microscope tunnel optique (Scanning Tunneling Optical Microscopy : STOM). Lillumination de l'objet peut aussi avoir lieu à travers une petite ouverture ou la fibre détectrice (nano-source).

Les images expérimentales obtenues nécessitent encore un important travail d'interprétation. De plus des études théoriques sont encore nécessaires pour essayer de déterminer le meilleur dispositif expérimental, pour une classe d'objets donnés. En effet, des problèmes importants restent à étudier : rôle de la nature et de la géométrie de la sonde, influence d'une métallisation de l'objet, comparaison des images obtenues par divers microscopes SNOM d'un même objet.

Un récent workshop a présenté les derniers résultats expérimentaux et fait le point sur les diverses approches théoriques [5]. M. Nevière et al. ont appliqué la théorie rigoureuse du réseau pour décrire des expériences de STOM. [6, 7]. Dereux et al. [8,9] utilisent le formalisme des fonctions de Green pour modéliser un microscope SNOM en transmission. Girard et al. ont adapté des modèles microscopiques, dérivés de la physique du solide, pour décrire diverses expériences en champ proche [10] et références incluses. Les méthodes précédentes nécessitent des moyens informatiques importants et sont non analytiques. Nous avons proposé un modèle macroscopique de STOM où le champ de diffracté était calculé de façon perturbative. Les calculs sont analytiques et permettent de comprendre aisément le rôle et l'influence des divers paramètres. En [11] nous avons discuté du problème de la résolution et du rôle de la distance pointe-surface. En [12] nous avons étudié l'influence du rayon de la pointe en utilisant un modèle multipolaire pour la sonde. En [13] nous avons comparé les images obtenues en utilisant des pointes dipolaires métallisées et diélectriques.

Récemment nous avons étendu notre méthode aux systèmes multicouches rugueux. Les rugosités sont le relief de l'objet, voire la pointe elle-même. Les rugosités sont supposées de hauteur faible par rapport à la longueur d'onde et une méthode perturbative permet de déterminer analytiquement les amplitudes des champs électromagnétiques diffractés dans chaque couche. Ce formalisme permet de modéliser des expériences de microscopie en champ proche avec une très large variété d'objet (objets multicouches, métallisés...) et des formes variées de pointes. Les divers types de microscopes (SNOM ou STOM en réflexion ou en transmission) peuvent aisément être décrits.

\section{Présentation du modèle}

Dans cet exposé, nous limiterons la discussion à des microscopes en champ proche utilisant une pointe pour sonder le champ de l'objet et où l'objet est éclairé par une onde plane en réflexion interne (STOM) ou à travers la pointe détectrice (SNOM). Mais notre méthode est applicable aux autres types de microscopes : ceux où l'éclairage ou la détection ont lieu par une petite ouverture $(30 \mathrm{~nm})$ dans un écran métallique. Lobjet sera ici monopériodique, de période $a$, bien que le modèle puisse s'appliquer à des objets non périodiques.

Le calcul du signal détecté consiste à déterminer le module carré du champ diffracté au niveau de la sonde. De fait nous ramenons le calcul d'une image SNOM à un problème de diffraction par un système multicouche (Fig. 1).

Le référentiel utilisé est indiqué sur la figure 2 , le motif du réseau est parallèle à $y$, l'axe $z$ est perpendiculaire au motif. Le plan d'incidence est le plan principal.

Les couches matérielles considérées constituent l'objet, son éventuel revêtement métallique, l'air avant la pointe, la pointe détectrice et le corps de la sonde. Chaque couche est caractérisée par sa constante diélectrique $\epsilon_{n}$, réelle pour un diélectrique, complexe pour un conducteur ou en cas d'absorption. 


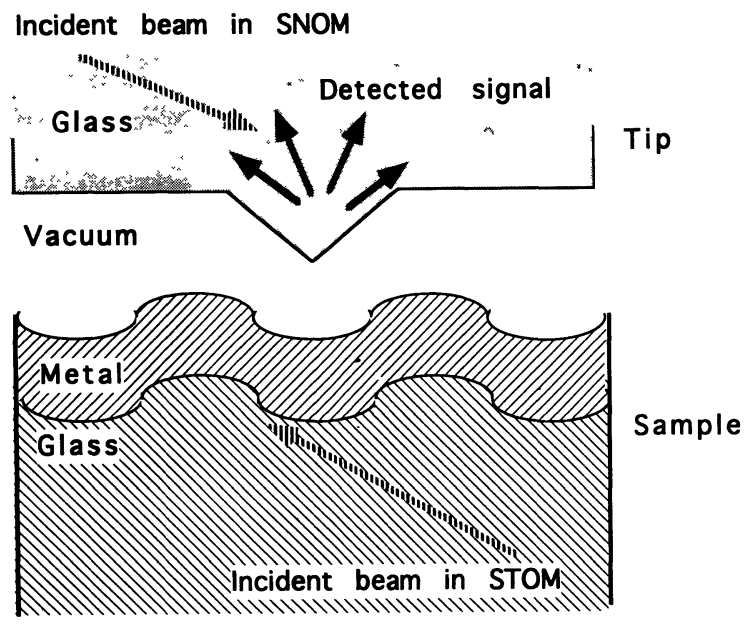

Fig. 1. - Principe de divers types de microscope optique en champ proche et modèle utilisé dans cet article. Léclairage peut être par réflexion interne (STOM) ou externe (SNOM par réflexion).

[Schematic of the multilayer structure used to model Near Field Optical Microscope principles' used in this paper. Illumination can be by transmission (STOM) or reflection (SNOM).]

Le développement de Rayleigh permet de développer le champ dans la couche $n$, en série d'ondes planes :

$$
\begin{aligned}
\mathbf{E}_{n}(x, y, z) & =\sum_{-\infty}^{+\infty} \mathbf{E}_{n} \uparrow(p) \exp \left[i u_{n}(p) x+i v_{n}(p) y+i w_{n}(p) z\right] \\
& +\sum_{-\infty}^{+\infty} \mathbf{E}_{n} \downarrow(p) \exp \left[i u_{n}(p) x+i v_{n}(p) y-i w_{n}(p) z\right]
\end{aligned}
$$

Les vecteurs d'onde des ordres diffractés dans le milieu $n$ vérifient les relations suivantes.

$$
\begin{gathered}
{\left[u_{n}(p)\right]^{2}+\left[v_{n}(p)\right]^{2}+\left[w_{n}(p)\right]^{2}=\epsilon_{n}\left(\frac{w}{c}\right)^{2} \text { (relation de dispersion) }} \\
u_{n}(p)=u_{0}+\frac{2 \pi p}{a}(\text { formule du réseau }) \\
v_{n}(p)=v_{0}
\end{gathered}
$$

où $u_{0}$ et $v_{0}$ sont les composantes selon $x$ et $y$ du vecteur d'onde du champ incident. Le champ dans chaque couche est composé de modes montants $\left(\mathbf{E}_{n} \uparrow(p)\right)$ en $\exp \left[i w_{n}(p) z\right]$ et descendants $\left(\mathbf{E}_{n} \downarrow(p)\right)$ en $\exp \left[-i w_{n}(p) z\right]$.

Si les surfaces séparant les milieux sont rigoureusement planes, seul subsiste l'ordre $p=0$. Les autres ordres $(p \neq 0)$ correspondent à de la diffraction sur le relief de l'objet ou sur la pointe détectrice. Les différents ordres peuvent être homogènes si $w_{n}(p)$ est réel ou évanescents si $w_{n}(p)$ est imaginaire pur. Les grandes fréquences spatiales de l'objet sont associées à des ordres de diffraction élevés $(|p|>>1)$ et correspondent à des ondes évanescentes. La résolution obtenue par les microscopes en champ proche est facilement expliquée si l'on admet que la sonde, balayant le champ diffracté à quelques nanomètres de distance, capte ces ondes évanescentes. 
Les inconnues du problème de diffraction sont les amplitudes $\mathbf{E}_{n} \uparrow(p)$ et $\mathbf{E}_{n} \downarrow(p)$ qui doivent être reliées à celle du champ incident. Les relations de continuité des champs permettent de résoudre formellement ce problème mais conduisent à un système linéaire infini qu'il faut résoudre numériquement. Dans notre approche nous supposons que chaque interface a une hauteur de corrugation faible par rapport à la longueur d'onde (relief de l'objet et taille de la pointe). Autrement dit, l'interface entre le milieu $n$ et le milieu $n+1$ a pour équation:

$$
z_{n}(x)=e_{n}+h_{n}(x)
$$

avec $h_{n}(x)<<\lambda$ mais l'épaisseur $e_{n}$ de la couche $n$ peut être quelconque.

La rugosité de l'interface $n \rightarrow n+1$ est développée sous forme d'une série de Fourier :

$$
h_{n}(x)=\sum_{-\infty}^{+\infty} \tilde{h}_{n}(p) \exp (i 2 \pi p x / a)
$$

Avec ces hypothèses, dans les équations aux limites, les exponentielles des équations des champs peuvent être développées :

$$
\exp \left[ \pm i w_{n}(p) z_{n}(x)\right] \cong \exp \left[ \pm i w_{n}(p) e_{n}\right]\left[1 \pm i w_{n}(p) h_{n}(x)\right]
$$

Cette approximation linéaire permet de déterminer les amplitudes des modes diffractés par une méthode de perturbation.

Si on néglige les rugosités de surface $\left(h_{n}(x)=0\right)$ on retrouve une relation matricielle entre les ordres $p=0$ de diffraction :

$$
\left[\begin{array}{l}
\mathbf{E}_{n} \uparrow(0) \\
\mathbf{E}_{n} \downarrow(0)
\end{array}\right]=\mathbf{M}_{n}^{0}\left[\begin{array}{l}
\mathbf{E}_{n-1} \uparrow(0) \\
\mathbf{E}_{n-1} \downarrow(0)
\end{array}\right]
$$

La relation précédante n'est autre que le formalisme matriciel bien connu (14) de détermination des amplitudes des champs transmis à travers un système multicouches. Le phénomène de diffraction est contenu dans les autres modes $p \neq 0$. En tenant compte des rugosités, la formule (7) injectée dans les conditions aux limites permet de déterminer ces ordres diffractés :

$$
\left[\begin{array}{l}
\mathbf{E}_{n} \uparrow(p) \\
\mathbf{E}_{n} \downarrow(p)
\end{array}\right]=\mathbf{M}_{n}^{d} \cdot\left[\begin{array}{l}
\mathbf{E}_{n-1} \uparrow(p) \\
\mathbf{E}_{n-1} \downarrow(p)
\end{array}\right]+\tilde{h}_{n}(p) \mathbf{V}_{n} \cdot\left[\begin{array}{l}
\mathbf{E}_{n-1} \uparrow(0) \\
\mathbf{E}_{n-1} \downarrow(0)
\end{array}\right](p \neq 0)
$$

Les matrices $\mathbf{M}_{n}^{d}, \mathbf{M}_{n}^{0}$ et $\mathbf{V}_{n}$ dépendent des composantes selon l'axe $z$ des vecteurs d'ondes des différents modes diffractés.

Les amplitudes diffractées $\mathbf{E}_{n} \uparrow(p)$ et $\mathbf{E}_{n} \downarrow(p)$ de l'harmonique $p$ dans la couche $n$ sont ainsi reliées linéairement aux amplitudes diffractées de même ordre dans la couche $n-1\left(\mathbf{E}_{n-1} \uparrow(p)\right.$ et $\left.\mathbf{E}_{n-1} \downarrow(p)\right)$ et également aux amplitudes transmises dans la même couche $\left(\mathbf{E}_{n-1} \uparrow(0)\right.$ et $\left.\mathbf{E}_{n-1} \downarrow(0)\right)$.

Les deux relations précédentes permettent de calculer de proche en proche toutes les amplitudes des modes diffractés dans toute les couches en fonction du champ incident. 


\section{Résultats}

Nous avons considéré un réseau diélectrique comme objet (en verre d'indice $n_{1}=1,5$ ) de période $a=200 \mathrm{~nm}$, de largeur $b=50 \mathrm{~nm}$ et de hauteur $c=10 \mathrm{~nm}$. Son profil est rectangulaire. Un tel réseau ne peut être résolu en microscopie classique et correspond approximativement à ceux étudiés par les expérimentateurs [3]. Le laser a une longueur d'onde de 632,8 nm. L'angle d'incidence est égal à $45^{\circ}$. Le plan d'incidence est le plan principal, perpendiculaire aux traits du réseau. Toutes les images de cet article sont calculées en polarisation T.M. qui donne pour cette géométrie la meilleure résolution [11].

La figure 2 présente les différents modèles de sonde que nous allons comparer. La sonde ponctuelle (Fig. 2a) peut être matérialisée par une petite sphère diélectrique ou métallique de rayon bien inférieur à la longueur d'onde et correspond expérimentalement à l'apex de la fibre optique taillée en pointe [3]. Dans ce cas, la sphère est décrite par sa polarisabilité dipolaire et elle est sensible au champ électrique en son centre. Le signal détecté est alors proportionnel au champ diffusé par la sphère, c'est à dire au module carré du champ au centre. Nous avons utilisé un tel modèle dans nos travaux précédants [11-13] et il reproduit assez bien certains résultats expérimentaux [15]. Nous avons effectué les calculs avec une pointe triangulaire (Fig. 2c) ou arrondie (Fig. 2d). Les deux pointes auront la même largeur et la même hauteur $(40 \mathrm{~nm})$. A des fins de comparaison nous avons aussi considéré une "sonde plane" sans aucune aspérité (Fig. 2b). Dans ces 3 derniers cas le signal détecté sera considéré comme égal au module carré du champ calculé juste au sommet de la pointe (points D des Figs. 2).
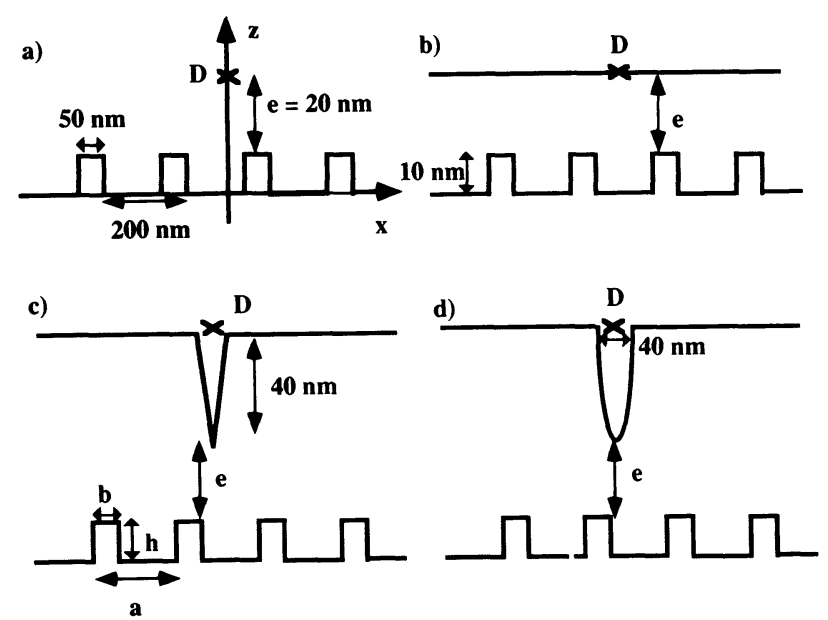

Fig. 2. - Divers modèles de sondes utilisés dans ce travail. a) sonde ponctuelle, b) sonde plane, c) sonde triangulaire, $\mathrm{d}$ ) sonde arrondie (elliptique). Dans tous les cas le point $\mathrm{D}$ où est calculé le module carré du champ détecté est indiqué. Lextrémité de la pointe est à $20 \mathrm{~nm}$ du sommet des traits du réseau. Dans les 4 cas, l'objet est un réseau diélectrique rectangulaire de période $200 \mathrm{~nm}$, de largeur $50 \mathrm{~nm}$ et de hauteur 10 nm.

[Four models of tip modeled. a) point tip, b) plane tip, c) triangular tip, d) round tip (elliptic). In all cases, the square modulus of the detected field is calculated at point $\mathrm{D}$. The sample is a rectangular grating of period $200 \mathrm{~nm}$, width $50 \mathrm{~nm}$, height $10 \mathrm{~nm}$. The distance between the end of the tip and the top of the grating is 20 nm.] 
Les premiers résultats (Figs. 3-5) présentés correspondent à des modèles de microscopes tunnel optique (STOM) où l'objet est éclairé en réflexion totale interne [3].

Les figures 3 présentent une comparaison des images calculées en utilisant les 4 modèles de sondes décrits ci-dessus et en déplaçant la sonde à une hauteur constante au dessus du plan du réseau. Pour avoir une comparaison efficace, la distance sonde objet est prise identique dans les 4 cas $(e=20 \mathrm{~nm})$ : soit pour la distance entre le bas de la sonde et le haut du motif de l'objet (sondes arrondies et triangulaires) soit entre le point $\mathrm{D}$ et le haut du motif pour les sondes ponctuelles ou plane.
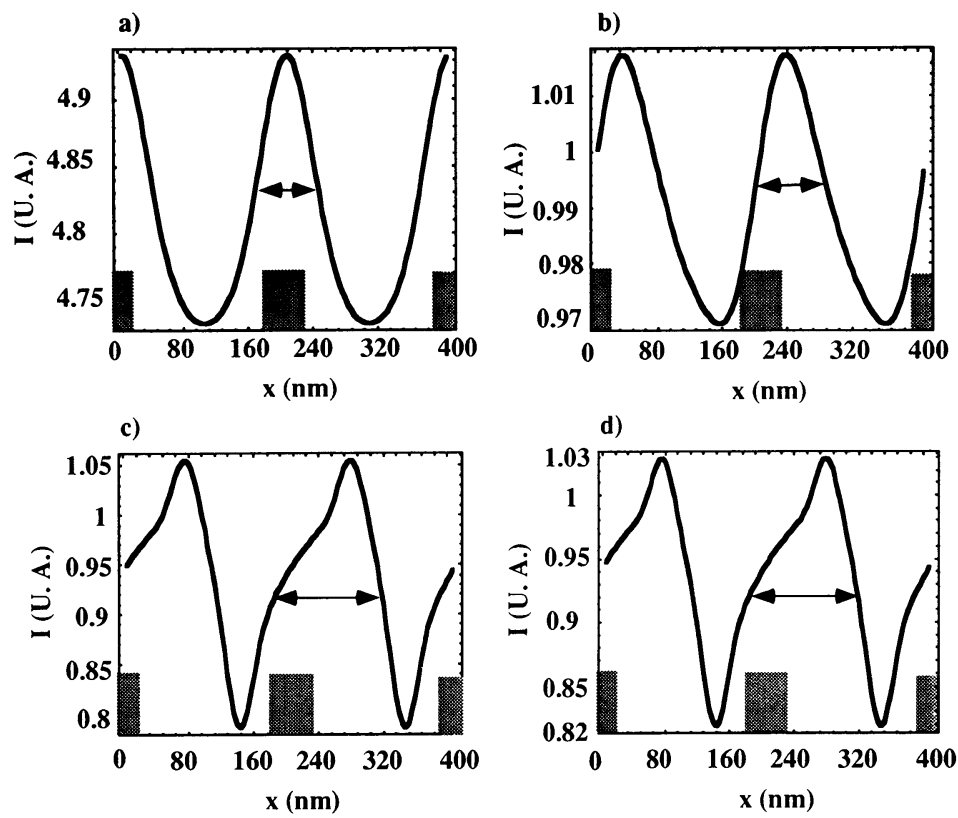

Fig. 3. - Profils d'intensité calculés avec les 4 modèles de sondes pour un Microscope Tunnel Optique (STOM). Les sondes sont représentées sur la figure 2. a) sonde ponctuelle b) Sonde plane c) Sonde triangulaire d) sonde arrondie. Léclairage a lieu en réflexion totale interne, dans le plan principal et avec un angle d'incidence de $45^{\circ}$. La longueur d'onde est $\lambda=632,8 \mathrm{~nm}$, la polarisation est T.M. Lobjet est un réseau diélectrique rectangulaire de période $200 \mathrm{~nm}$, de largeur $50 \mathrm{~nm}$ et de hauteur $10 \mathrm{~nm}$. Le point de détection est à $20 \mathrm{~nm}$ du sommet des traits du réseau.

[Intensity profiles calculated for STOM with 4 tip geometries. Tips are described in figure 2. a) point tip, b) plane tip, c) triangular tip, d) round tip. The angle of incidence is $45^{\circ}$, (total internal reflection), the plane of incidence is perpendicular to the grooves. The wavelength is $632,8 \mathrm{~nm}$. The polarization is T.M. The sample is a dielectric rectangular grating with period $200 \mathrm{~nm}$, width $50 \mathrm{~nm}$ and height $10 \mathrm{~nm}$. The detection is at 20 $\mathrm{nm}$ above the grating tracks.]

Pour la figure 3a, la sonde est ponctuelle : on représente le module carré du champ proche diffracté par l'objet. Le point où l'on calcule le champ se déplace avec la sonde au dessus du réseau à une hauteur constante de $20 \mathrm{~nm}$ au dessus des traits. La courbe ainsi obtenue reproduit assez bien le profil de l'objet mais l'amplitude des hautes harmoniques a déjà été fortement atténuée. Une étude plus complète de ces effets de filtrage du spectre spatial de l'objet en fonction de la polarisation et de la distance est présenté dans les références [11] et [15]. 
Avec la sonde plane, l'image obtenue est déformée (Fig. 3b) en raison de la frustration des ondes évanescentes diffractées par l'objet. La hauteur de détection est la même mais, avec cette sonde, les harmoniques élevées ont une importance plus faible dans le signal détecté.

Avec les sondes triangulaire ou arrondie, les images calculées (respectivement Figs. 3c et 3d) comportent des fréquences spatiales élevées. Les deux courbes sont assez semblables mais comportent néanmoins des différences en ce qui concerne la dynamique et donc la reconstitution des flancs raides. La sonde triangulaire (Fig. 3c) donne une meilleure dynamique de l'image : la visibilité de l'image est de $13,5 \%$ dans ce cas. Par contre, pour une sonde arrondie (Fig. $3 \mathrm{~d}$ ), la visibilité tombe à $10 \%$. La visibilité est le rapport de la différence des intensités maximales et minimales à leur somme. Ce résultat permet de conclure que la forme de l'extrémité de la sonde a une grande importance dans le signal détecté en STOM. Ces deux dernières figures sont différentes de celle obtenue avec le détecteur ponctuel. En effet la sonde et le relief de l'objet sont couplés et le signal détecté est le champ diffracté par l'ensemble pointe- objet. Le spectre spatial du signal obtenu comporte aussi bien des renseignements sur la pointe que sur l'objet. La largeur des pics à mi hauteur dans les images avec sonde correspond en conséquence à la largeur de l'objet plus le double de la largeur de la sonde (130 nm environ). Nous avons choisi la largeur à mi-hauteur car, dans le cas du détecteur ponctuel elle est approximativement égale à la largeur de l'objet (Fig. 3a). Cet effet d'élargissement dans les profils $3 c$ et $3 d$ est dû à la sonde ; la limite de résolution de ce type de microscopie semble donc dépendre surtout de la taille de l'extrémité de la sonde.

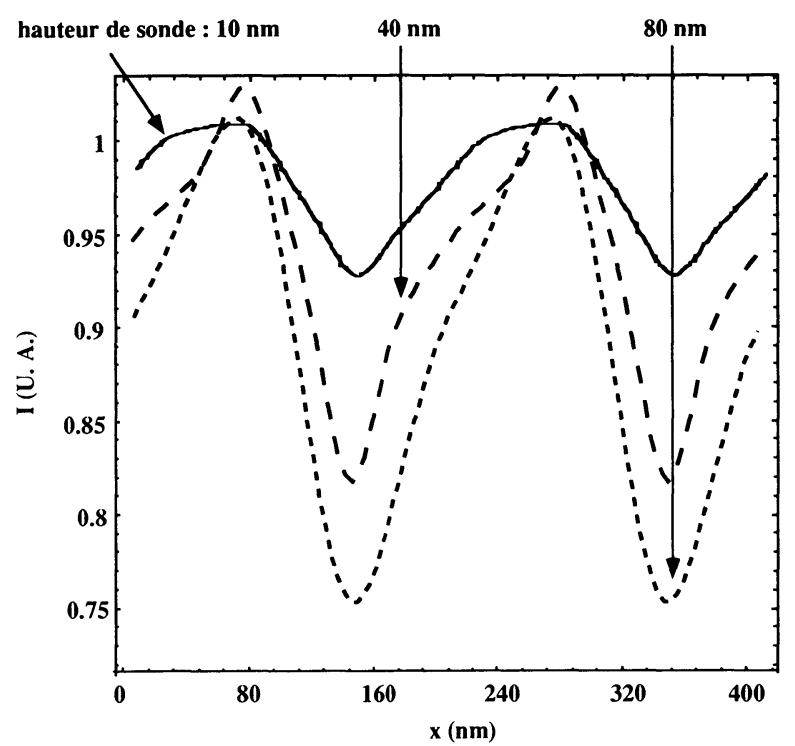

Fig. 4. - Profils d'intensité calculés en microscopie STOM avec deux sondes triangulaire de hauteur différente mais de même largeur $40 \mathrm{~nm}$. En trait plein : sonde de hauteur $10 \mathrm{~nm}$ et en pointillés: sondes de hauteur $40 \mathrm{~nm}$ et $80 \mathrm{~nm}$. L'éclairage et l'objet sont décrits en figure 3 .

[Intensity profiles calculated in STOM with three heights for a triangular tip. The width of tips is always 40 $\mathrm{nm}$. Solid line: the tip height is $10 \mathrm{~nm}$, dashed lines: the tip height is $40 \mathrm{~nm}$ and $80 \mathrm{~nm}$. Lighting and sample are described in figure 3.]

La figure 4 présente l'influence de la hauteur de la pointe sur l'allure de l'image. Les deux courbes correspondent à une pointe triangulaire. Les courbes en pointillés correspondent à une 
hauteur de la pointe égale à $40 \mathrm{~nm}$ puis $80 \mathrm{~nm}$. La courbe pleine est obtenue pour une pointe de $10 \mathrm{~nm}$ de haut. En allongeant ainsi la pointe on constate d'importantes variations des courbes calculées. Cette grande sensibilité des images aux variations des paramètres expérimentaux (Figs. 3c, 3d, 4) est aussi observée expérimentalement. De plus la meilleure reconstitution des hautes harmoniques pour des pointes fines, de faible ouverture est aussi confirmée par d'autres modélisations [15].

La méthode décrite ci-dessus permet de traiter des objets de forme variée et comme il s'agit d'un formalisme capable de traiter un système multicouches le cas où l'objet est recouvert d'une fine couche métallique est très facilement intégré dans nos calculs. Nous avons ainsi étudié l'influence d'un mince dépôt métallique.

Les figures 5 comparent les images du même réseau en Microscopie Tunnel Optique. Les conditions d'éclairage sont les mêmes, la sonde est triangulaire (Fig. 2c) dans les deux cas, mais dans le premier cas (Fig. 5a) l'objet est nu, dans le second (Fig. 5b) il est recouvert d'une couche d'argent de 12,8 nm d'épaisseur. On suppose que cette couche d'argent reproduit parfaitement la forme du profil du réseau en verre. Les deux courbes ne sont pas similaires en raison de la résonance plasmon due à la couche métallique. Cette déformation n'apparaît pas pour des conditions d'éclairage différentes (polarisation TE...) Par contre on constate une augmentation de la visibilité de l'image : de $27 \%$ à $32 \%$. Une telle métallisation légère de l'objet à "imager" pourrait être utile expérimentalement pour augmenter le rapport signal sur bruit.

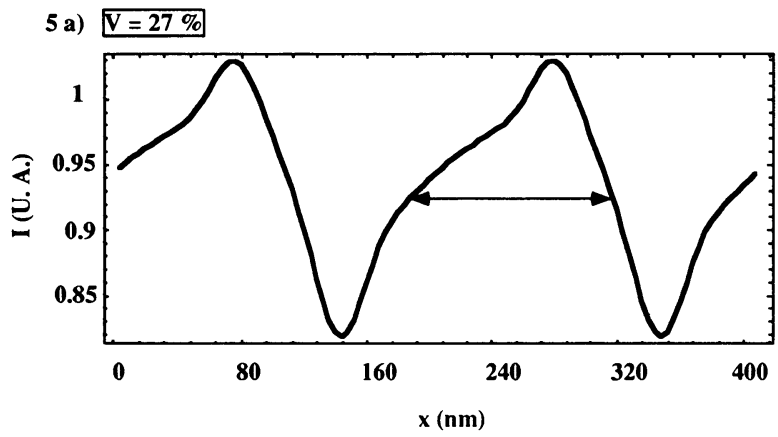

5b) $V=32 \%$

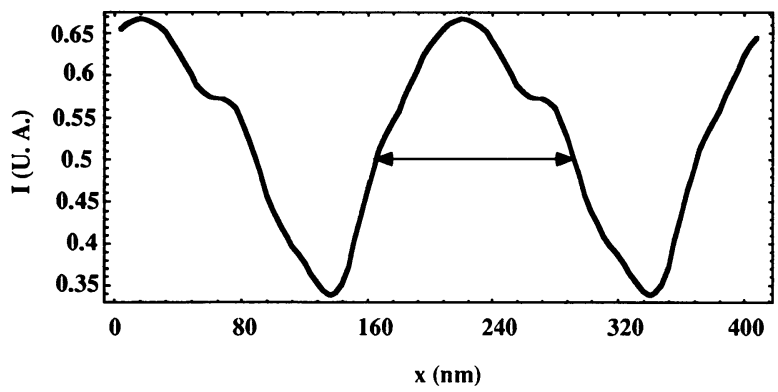

Fig. 5. - Comparaison des profils d'intensité calculés en Microscopie Tunnel Optique avec la pointe triangulaire définie en (2c). a) : objet non métallisé. b) : objet recouvert d'une couche d'argent de 12,8 $\mathrm{nm}$ d'épaisseur. L'éclairage et l'objet sont décrits en figure 3.

[Influence of sample coating, comparison between intensity profiles calculated in STOM with triangular tip (Fig. 2c). a): glas sample. b): the same sample coated with $12.8 \mathrm{~nm}$ of silver. Lighting and sample are described in figure 3.] 
Pour conclure cette rapide présentation de résultats, la figure 6 présente une image théorique du même objet que ci-dessus mais pour un microscope en champ proche fonctionnant en réflexion où l'objet est éclairé par la pointe qui sert à la détection (SNOM par réflexion). Cette configuration est essentiellement développée par M. Spajer à Besançon $[16,17]$. La pointe est encore la pointe triangulaire (Fig. 2c). Pour ce type de microscope (et les paramètres choisis) l'image théorique obtenue est assez différente de celle calculée en configuration STOM (comparer la Fig. 3c à la Fig. 5), l'image obtenue paraît translatée par rapport à l'objet, sans doute par des effets d'interférence entre les modes diffractés par la structure. Elle fait de plus apparaître un apparent doublement de période. La distance entre les deux pics centraux du motif superposé à l'objet est, comme dans le cas du STOM, influencée par la taille latérale de la sonde (environ $130 \mathrm{~nm}$, soit de l'ordre de la somme de la taille latérale de l'objet et du double de celle de la sonde).

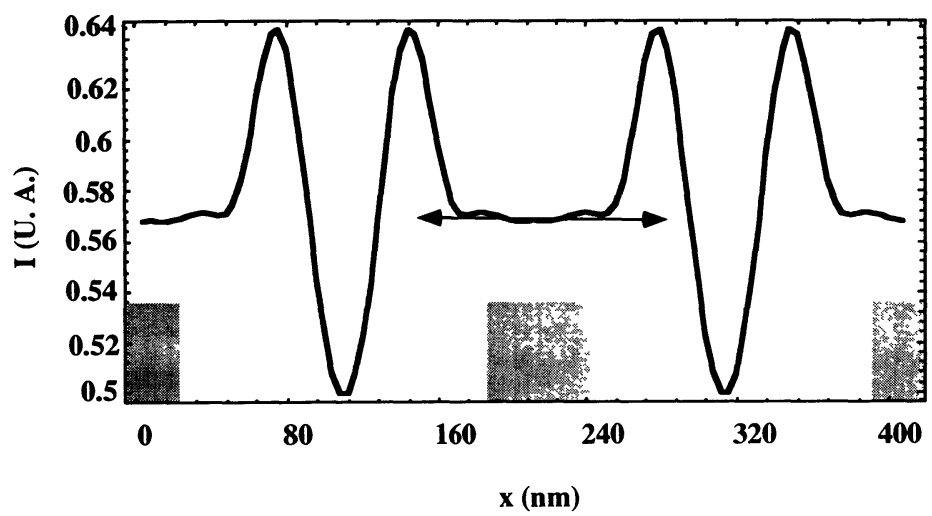

Fig. 6. - Profil d'intensité d'un réseau carré calculé en Microscopie Champ proche par réflexion. Lobjet est décrit figure 3. Mais ici il est éclairé par la pointe détectrice avec un angle d'incidence de $0^{\circ}$.

[Intensity profiles of a rectangular grating, calculated for SNOM in reflection configuration. The sample is described in figure 3. But it is illuminated through the tip. The angle of incidence is $0^{\circ}$.]

\section{Conclusion}

Dans cet article nous avons décrit le principe d'une méthode permettant de modéliser les microscopes optique en champ proche. Elle est basée sur un calcul perturbatif des champs diffractés par un système multicouches rugueuses. Nous avons présenté quelques résultats en microscopie tunnel optique (STOM) et en SNOM par réflexion. Nous avons comparé les images obtenues par divers modèles de pointes. Nous avons montré que notre modèle permet de calculer des images pour des objets métallisés. Nous avons appliqué cette méthode aux microscope STOM et SNOM en réflexion utilisant une fibre optique taillée en pointe. Comme nous utilisons une méthode perturbative, la hauteur des relief et de la pointe doit être petite.

La théorie rigoureuse du réseau $([6,18]$ et références incluses) permet, d'étudier des pointes de hauteur quelconque. Mais les temps de calcul et les problèmes de convergence peuvent être rédhibitoires. 
En microscopie Optique en Champ Proche seule l'apex de la sonde semble jouer un rôle important ce qui justifie l'emploie de la méthode perturbative. Elle est facilement applicable à des objets tridimensionnels périodiques ou non périodiques. Elle permet aussi de modéliser les autres types de microscopes SNOM qui utilisent une petite ouverture comme source ou détecteur [1,2].

\section{References}

[1] Pohl D., "Scanning near-field optical microscopy" in Advances in Optical and Electron Microscopy, C.R.J. Sheppard and T. Mulvey Eds. (Academic Press, Londre, 1990).

[2] Betzig E., Trautman J.K., Harris T.D., Weiner J.S., Kostelar R.L., "Breaking the diffraction barrier: optical microscopy on a nanometric scale", Science 251 (1991) 1468-1470.

[3] Courjon D., Bainier C., Spajer M., "Imaging of submicron index variations by scanning optical tunneling", J. Vac. Sci. Technol. B 10 (1992) 2436.

[4] Near Field Optics, D. Pohl and D. Courjon Eds. (Kluwer Acad., 1993).

[5] Van Labeke D., Barchiesi D., “Theoretical problems in Scanning Near-field Optical Microscopy”, Near Field Optics, D. Pohl and D. Courjon Eds. (Kluwer Acad., 1993) 157-178.

[6] Nevière M., Vincent P., "Diffraction gratings as components for photon scanning tunneling image interpretation", Near Field Optics, D. Pohl and D. Courjon Eds. (Kluwer Acad., 1993) 377-379.

[7] Goudonnet J.P., Salomon L., De Fornel F., Bourillot E., Adam P., Nevière M., "Recent progress in photon scanning tunneling microscopy", Scanning probe Microsc. (SPIE Proc. 1992) 12-23.

[8] Dereux A., Pohl D., "The $90^{\circ}$ prism as a model SNOM probe: near-field, photon tunneling and farfield properties", Near Field Optics, D. Pohl and D. Courjon Eds. (Kluwer Acad., 1993) 189-198.

[9] Dereux A., Vigneron J.P., Lambin Ph., Lucas A.A., "Theory of near-field optics with applications to SNOM and optical binding", Physica B 175 (1991) 65-67.

[10] Girard C., Bouju X., Dereux A., "Optical near-field detection and local spectroscopy of a surface: a self consistent theoretical study", Near Field Optics, D. Pohl and D. Courjon Eds. (Kluwer Acad., 1993) 199-208.

[11] Van Labeke D., "Scanning tunneling optical microscopy: a theoretical macroscopic approach", J. Opt. Soc. Am. A9 (1992) 732-739.

[12] Barchiesi D., Van Labeke D., "Application of Mie scattering of evanescent waves to scanning optical tunneling microscopy theory", J. Mod. Opt. 40 (1993) 1239-1254.

[13] Van Labeke D., Barchiesi D., "Probes for scanning tunneling optical microscopy: a theoretical comparison", J. Opt. Soc. Am. A10 (1993) 2193-2201.

[14] Lekner J., "Theory of reflection", Martinus Nijhoff Publishers (Dordrecht 1987) p. 221 et toutes les références inclues.

[15] Barchiesi D., "Modélisation des microscopes optiques en champ proche : STOM, SNOM. Optimisation et spectroscopie", thèse, Université de Besançon (1993).

[16] Courjon D., Vigoureux J.M., Spajer M., Sarayeddine K., Leblanc S., "External and internal reflection near-field microscopy: experiments and results", App. Opt.(1990) 3734-3740.

[17] Jalocha A., "Etude d'un microscope optique à sonde locale en réflexion. Réalisation et caractérisation des sondes utilisées", thèse (Université de Besançon, 1993).

[18] Maystre D., "Rigorous grating theory of diffraction gratings", Progress in Optics XXI, E. Wolf Ed. (Elsevier, Amsterdam, 1984). 